\title{
ACCURACY OF TRANSTHORACIC ECHOCARDIOGRAPHY IN DIAGNOSIS OF CARDIAC MYXOMA: SINGLE CENTRE EXPERIENCE
}

\author{
Katja Prokšelj ${ }^{1}$, Polona Kačar ${ }^{1}$, Nejc Pavšič ${ }^{1}$, Mojca Bervar ${ }^{1}$, and Zvezdana Dolenc \\ Stražar ${ }^{2}$ \\ ${ }^{1}$ Department of Cardiology University Medical Center Ljubljana Zaloska cesta 71525 \\ Ljubljana Slovenia \\ ${ }^{2}$ Univerza v Ljubljani Medicinska Fakulteta
}

December 20, 2021

\begin{abstract}
The differential diagnosis of cardiac myxomas $(\mathrm{CM})$, the most common benign primary cardiac tumors, is broad and a thorough diagnostic workup is required to establish accurate diagnosis prior to surgical resection. Transthoracic echocardiography (TTE) is usually the first imaging modality used for diagnosis of suspected CM. Purpose In a single tertiary centre study, we sought to determine the accuracy, sensitivity, and specificity of TTE in the diagnosis of CM and to determine echocardiographic characteristics indicative of CM. Methods and results We retrospectively analyzed clinical, echocardiographic, and pathohistological findings of 73 patients consecutively admitted for suspected CM. After diagnostic workup, 53 (73\%) patients were treated surgically at our institution. Based on preoperative TTE, patients were divided into a CM group ( $\mathrm{n}=45,85 \%)$ and non-myxoma (NM) group. Of the 53 pathohistological specimens obtained during surgery, 39 (73\%) were CM. The sensitivity and specificity of preoperative echocardiography were $97 \%$ and $50 \%$, respectively. The overall accuracy was $85 \%$. All NM tumors were found in an atypical location and $72 \%$ of $\mathrm{CM}$ were found in a typical position in the left atrium $(\mathrm{p}<0.001)$. Tumors in NM group were significantly smaller than CM $(24.3 \pm 13.2 \mathrm{~mm}$ vs $37.9 \pm 18.3 \mathrm{~mm}, \mathrm{p}=0.017)$. Conclusion Our study confirms very good accuracy of TTE in the diagnosis of CM. The most important echocardiographic characteristics to differentiate between CM and tumors of different etiology are tumor location and size. Smaller tumors presenting at an atypical location are less likely to be diagnosed as CM, and these require additional imaging modalities for accurate diagnosis.
\end{abstract}

\section{ACCURACY OF TRANSTHORACIC ECHOCARDIOGRAPHY IN DIAGNOSIS OF CAR- DIAC MYXOMA: SINGLE CENTRE EXPERIENCE}

Polona Kačar, $\mathrm{MD}^{\mathrm{a}}$, Nejc Pavšič, MD ${ }^{\mathrm{a}}$, Mojca Bervar, MD, $\mathrm{PhD}^{\mathrm{a}}$, Zvezdana Dolenc Stražar, $\mathrm{MD}^{\mathrm{b}}$, assist. prof. Katja Prokšelj, MD, PhD ${ }^{\mathrm{a}, \mathrm{b}^{*}}$

Department of Cardiology, University Medical Center Ljubljana, Zaloska cesta 7, 1525 Ljubljana, Slovenia

Faculty of Medicine, University of Ljubljana, Vrazov trg 2, 1000 Ljubljana

*Corresponding author: Katja Prokšelj, katja.prokselj@gmail.com, tel. +(386)1-522-8572, fax. +(386)1-5222828

\section{Abstract}

The differential diagnosis of cardiac myxomas (CM), the most common benign primary cardiac tumors, is broad and a thorough diagnostic workup is required to establish accurate diagnosis prior to surgical resection. 
Transthoracic echocardiography (TTE) is usually the first imaging modality used for diagnosis of suspected CM.

\section{Purpose}

In a single tertiary centre study, we sought to determine the accuracy, sensitivity, and specificity of TTE in the diagnosis of CM and to determine echocardiographic characteristics indicative of CM.

\section{Methods and results}

We retrospectively analyzed clinical, echocardiographic, and pathohistological findings of 73 patients consecutively admitted for suspected CM. After diagnostic workup, $53(73 \%)$ patients were treated surgically at our institution. Based on preoperative TTE, patients were divided into a CM group $(\mathrm{n}=45,85 \%)$ and non-myxoma (NM) group. Of the 53 pathohistological specimens obtained during surgery, 39 (73\%) were CM. The sensitivity and specificity of preoperative echocardiography were $97 \%$ and $50 \%$, respectively. The overall accuracy was $85 \%$. All NM tumors were found in an atypical location and $72 \%$ of CM were found in a typical position in the left atrium $(\mathrm{p}<0.001)$. Tumors in NM group were significantly smaller than CM $(24.3 \pm 13.2 \mathrm{~mm}$ vs $37.9 \pm 18.3 \mathrm{~mm}, \mathrm{p}=0.017)$.

\section{Conclusion}

Our study confirms very good accuracy of TTE in the diagnosis of CM. The most important echocardiographic characteristics to differentiate between CM and tumors of different etiology are tumor location and size. Smaller tumors presenting at an atypical location are less likely to be diagnosed as CM, and these require additional imaging modalities for accurate diagnosis.

Keywords: cardiac mass; cardiac myxoma; cardiovascular imaging; echocardiography

\section{Introduction}

Although rare, cardiac myxoma (CM) represents the most common benign primary cardiac tumor[1]. Many patients are asymptomatic and CM is often an incidental finding[2]. Potentially life-threatening complications such as tumor obstruction or embolization can occur, making accurate diagnosis crucial $[3,4]$. However, diagnosis is challenging due to the broad differential diagnosis of $\mathrm{CM}$, which includes other cardiac tumors and cardiac masses such as thrombi, vegetations, calcific lesions, and other rare conditions.

Transthoracic echocardiography (TTE) nowadays represents the most commonly used initial imaging modality in the diagnostic workup of CM. It provides information on tumor size, location, attachment point, morphology, mobility, and its relation to surrounding structures. The majority of CM are located in the left atrium, attached to the atrial septum in the region of the fossa ovalis. These are considered as typical CM, but atypical localizations outside the left atrium have been described in around 30\% [5]. Size and appearance (solid and round or polypoid) may also vary considerably in CM [6].

TTE has an excellent detection rate for CM and a sensitivity of 90-96\% in diagnosing CM has been reported [4]. However, the heterogeneous morphological presentation leads to overlap with other cardiac masses and may affect the specificity and accuracy of TTE in CM diagnosis. Furthermore, TTE lacks tissue characterization [7]. Multimodality cardiac imaging ensures a more detailed analysis. Ultimately, the final diagnosis is made by histopathological examination of the excised tumor [8].

The aim of our single-center study was to evaluate the utility and accuracy of TTE in the diagnosis of CM and to determine echocardiographic characteristics indicative of pathohistologically confirmed CM.

\section{Methods}

We retrospectively analyzed clinical, echocardiographic, and pathohistological findings of all consecutive adult patients ([?]18 years of age) referred to our Department of Cardiology for suspected CM between 2005 and 2020 . 
All patients had TTE performed as part of the standard diagnostic workup. Echocardiographic characteristics of the cardiac mass were obtained, including mass location, surface (smooth or lobulated vs. villous) and appearance (homogenous vs. heterogenous). The mobility of the mass and the presence or absence of obstruction were also noted. Based on TTE findings, patients were diagnosed with either CM, other nonmyxomal (NM) cardiac tumor, or cardiac masses of other etiology (thrombus, infective endocarditis, etc.). Diagnosis of CM was made individually by the cardiologist performing TTE based on typical morphological characteristics of the cardiac mass. In some cases, additional imaging methods were used, either due to poor TTE acoustic windows or atypical tumor presentation. TTE contrast imaging was not performed in any of the cases.

Patients with CM or NM cardiac tumors were referred for surgery and pathohistological samples of the tumors were collected and analyzed to determine the final diagnosis. The accuracy, sensitivity, and specificity of TTE were determined by comparing echocardiographic and pathohistological diagnosis. Furthermore, echocardiographic characteristics of pathohistologically proven CM were compared to NM cardiac tumors. The study was approved by the national ethics committee and it has been carried out in accordance with the Declaration of Helsinki.

\section{Statistical analysis}

Continuous variables are presented as mean +- standard deviation and categorical variables as numbers and percentage. The independent Student's t-test was used to compare continuous variables. Categorical variables were analyzed using the $\chi^{2}$ test. The sensitivity, specificity, negative predictive value, and positive predictive value of echocardiographic diagnosis of CM were calculated using the results of pathohistological examination as the gold standard. Accuracy was determined as the sum of true negative and positive tests divided by all tests. All statistical analyzes were performed using SPSS version 26.0 software. Values of $\mathrm{p}<0.05$ were considered statistically significant.

\section{Results}

\section{Baseline characteristics}

During the 15-year period, 73 patients were referred for evaluation of suspected CM. All patients underwent TTE and $63(86 \%)$ were diagnosed with CM or NM cardiac tumor. Of the remaining 10 (14\%) patients, five were diagnosed with thrombus and were treated accordingly with anticoagulation therapy. In three cases pseudotumor was diagnosed; one had a prominent Eustachian valve, one had a prominent Chiari network, and one had lipomatous hypertrophy of the interatrial septum. In two patients no obvious cardiac mass was found on repeat TTE.

In 56 cases (77\%) additional imaging techniques were used to confirm the diagnosis, either due to suboptimal image quality on TTE or atypical tumor presentation. CMR was used most frequently $(\mathrm{n}=25,34 \%)$, followed by TEE $(n=24,33 \%)$ and CT $(n=5,7 \%)$. In two patients PET-CT was performed to detect possible distant metastases. No working diagnosis changed after additional imaging techniques.

After complete diagnostic workup, 53 (84\%) of the 63 patients diagnosed with either CM or NM were referred for surgery at our institution. One patient underwent surgery at another institution and was not included in further analysis. The remaining 9 patients did not undergo surgery, either due to poor clinical condition $(n=4)$ or patient's choice $(n=1)$. In four patients the mass was very small and periodic TTE follow-up was indicated.

The mean age of the operated patients was $64 \pm 14$ years (26-85 years), 35 patients (66\%) were female. The most common complaint was dyspnea (18 patients, 34\%), followed by embolic events in 8 patients (15\%), chest pain in 5 patients (9\%), constitutional signs in 3 patients (6\%) and palpitations in 2 patients (4\%). Seventeen patients (32\%) were asymptomatic. The mass was an incidental finding in 23 patients (43\%), most commonly on TTE (61\%) and chest CT (39\%) performed for other indication.

Echocardiographic characteristics 
Based on preoperative echocardiographic findings, the 53 operated patients were divided into two groups: a CM group (45 patients, 85\%) and a NM group (8 patients, 15\%). Preoperative echocardiographic characteristics are depicted in Table I. All tumors were solitary. The mean tumor size in the CM group was $35.3 \pm 18.6 \mathrm{~mm}$ (range: $10-81 \mathrm{~mm}$ ). The majority of CM were located in the atria; $80 \%$ in the left atrium and $18 \%$ in the right atrium. One tumor was found in the left ventricle. Tumors found in the left atrium were most frequently attached to the atrial septum in the region of the fossa ovalis $(n=27,75 \%)$ (Figure 1$)$. Other attachment sites in the left atrium included the mitral valve $(n=4$, two were attached to the posterior leaflet, one to the anterior leaflet, and one to the posterior annulus of the mitral valve), other areas of the atrial septum $(\mathrm{n}=3$, posterior part of the atrial septum), the free atrial wall $(\mathrm{n}=1)$ and left atrial appendage $(\mathrm{n}=1)$. The tumors were mostly mobile $(\mathrm{n}=32,71 \%)$. Mitral valve obstruction was observed in $10(22 \%)$ patients and tricuspid valve obstruction in $1(2 \%)$ patient.

The mean tumor size in the NM group was $30.3 \pm 16.9 \mathrm{~mm}$ (range: 8-50 mm) (Table I). All NM tumors were found in an atypical location, most frequently in the right atrium $(\mathrm{n}=4,50 \%)$. Two were attached to the left atrial free wall or posterior mitral valve leaflet and one to the aortic valve.

TTE sensitivity, specificity, and accuracy analysis

Pathohistological samples were obtained from the resected tumors in all 53 surgical procedures performed at our institution. Pathohistological evaluation confirmed CM in 39 of 53 operated patients (73\%) (Figure 1).

The calculated sensitivity and specificity of preoperative echocardiography were $97 \%$ and $50 \%$, respectively. The overall accuracy of TTE in diagnosing CM was 85\% (Figure 2). In 7 patients (13\%) diagnosed as CM on preoperative TTE, pathohistology revealed different NM cardiac tumors: papillary fibroelastoma in 5 cases, one case of angioleiomyoma and one malignant melanoma metastasis. Only one patient preoperatively classified as NM cardiac tumor had CM.

Pathohistologically confirmed NM cardiac tumors were significantly smaller than CM $(24.3 \pm 13.2 \mathrm{~mm}$ vs $37.9 \pm 18.3 \mathrm{~mm}, \mathrm{p}=0.017$ ) (Table II). There was also a statistically significant difference in tumor location between the two groups. All NM tumors were located at an atypical position (seven in the right atrium, five in the left atrium but at an atypical site and two in the left ventricle) and $72 \%$ of CM were found at the typical location within the left atrium $(\mathrm{p}<0.001)$. The calculated sensitivity and specificity of tumor location in diagnosis of myxoma was $100 \%$ and $56 \%$, respectively. There was no significant difference in other demographic (age, sex) or echocardiographic characteristics (mobility, surface, appearance) between groups.

\section{Discussion}

Our single-center study confirms very good overall accuracy of TTE in CM diagnosis. This is clinically important as accurate assessment of cardiac masses is essential for appropriate clinical management and treatment of these patients.

Diagnosis of CM can be challenging since patients are frequently asymptomatic or have only non-specific signs and symptoms. Dyspnea, a frequent and non-specific symptom of cardiac disease, was the most common complaint in our CM group, which is consistent with previous reports [9-11]. Clinical presentation itself rarely suggests the diagnosis of $\mathrm{CM}$; therefore, cardiac imaging is essential in the evaluation of patients with suspected CM. Echocardiography is the most widely used imaging modality that provides important information about the location, size, and appearance of the cardiac mass, as well as possible complications (e.g. obstruction). Previous studies have shown that CM are typically solitary, located in the left atrium, smooth in surface and mobile $[12,13]$. However, the morphological presentations of CM are often atypical and heterogeneous, leading to overlap with other NM cardiac tumors and cardiac masses.

The results of our study show very good overall accuracy (85\%) of TTE in CM diagnosis with excellent sensitivity (97\%). However, the specificity of TTE is modest (50\%) and caution is warranted as misdiagnosis of CM is possible. In our study, 5 of the misdiagnosed cases of CM were actually papillary fibroelastoma, which is also a common primary benign cardiac tumor. One of the suspected CM was actually a metastasis 
of malignant melanoma, underlying the importance of surgical excision and pathohistological examination of all suspected CM.

According to our results tumor localization and tumor size are the best echocardiographic characteristics to distinguish between CM and NM cardiac tumors. CM are typically located in the left atrium attached to the interatrial septum at the region of fossa ovalis, which was also shown in our study [14]. In our patients, $72 \%$ of CM were located typically. However, all tumors preoperatively misdiagnosed as CM were located in atypical locations, such as the right atrium and left ventricle. Tumors in the NM group were also significantly smaller compared to tumors in the CM group. However, there was no significant difference in age, sex, and other echocardiographic characteristics (mobility and surface) between the groups.

The differential diagnosis of CM is broad and definite diagnosis is crucial, as treatment varies depending on the diagnosis. Multimodality cardiac imaging improves the diagnostic accuracy of different cardiac masses. In the majority of our patients, at least one additional imaging modality was used as a part of the diagnostic workup. TEE improves image quality and provides more morphological information than TTE [15]. Computed tomography and cardiac magnetic resonance provide additional information on topographic relationships and tissue characteristics, and may detect other pathological conditions within the thorax [16,17]. Assessment of cardiac tumors by CMR is more accurate than echocardiography and can reliably distinguish between benign and malignant cardiac tumors [18-20].

There are some limitations to this study. First, this is a retrospective study with a relatively small study population. However, the population size is comparable to other studies on CM. Due to the low incidence of cardiac tumors, only multicenter studies can provide a larger scale patient population. Second, preoperative echocardiography was performed by different echocardiographers, potentially exposing the results to inter-investigator variability in determining the diagnosis. Due to the study inherently including participants already given a working diagnosis of CM, any cardiologist performing TTE was likely influenced by the information provided upon referral. A larger, multicenter, prospective study could serve to identify echocardiographic and clinical characteristics specific to CM, as well as other cardiac tumors, further increasing the utility of preoperative diagnostic modalities.

\section{Conclusions}

TTE is very accurate in diagnosing CM. Tumor localization and size are the most important echocardiographic characteristics that can differentiate between CM and NM. The diagnosis of CM is less likely in atypical tumor location and smaller tumor size. In such cases, caution is advised and other non-invasive imaging modalities, such as CMR or CT, should be performed to confirm the diagnosis.

\section{Author contributions}

The concept of this article was designed by Katja Prokšelj. Nejc Pavšič and Polona Kačar collected the data and wrote the draft of the article. Polona Kačar analyzed the data. Critical revision of the article was made by Mojca Bervar, Zvezdana Dolenc Stražar and Katja Prokšelj.

\section{Declarations}

Conflicts of interest: The authors declare that they have no conflict of interest.

\section{References}

1. Roberts WC: Primary and secondary neoplasms of the heart. Am J Cardiol 1997:80:671-682.

2. Karabinis A, Samanidis G, Khoury M, et al: Clinical presentation and treatment of cardiac myxoma in 153 patients. Medicine (Baltimore) 2018:97:e12397-e12397.

3. Lee S, Kim J-H, Na C-Y, et al: Eleven Years' Experience with Korean Cardiac Myxoma Patients: Focus on Embolic Complications. Cerebrovasc Dis 2012:33:471-479. 
4. Samanidis G, Khoury M, Balanika M, et al: Current challenges in the diagnosis and treatment of cardiac myxoma. Kardiol Pol 2020:78:269-277.

5. Poterucha TJ, Kochav J, O'Connor DS, et al: Cardiac Tumors: Clinical Presentation, Diagnosis, and Management. Curr Treat Options Oncol 2019:20:1-15.

6. Tyebally S, Chen D, Bhattacharyya S, et al: Cardiac Tumors JACC CardioOncology State-of-the-Art Review. Jacc Cardiooncology 2020:2:1-19.

7. Colin GC, Gerber BL, Amzulescu M, et al: Cardiac myxoma: a contemporary multimodality imaging review. Int J Cardiovasc Imaging 2018:34:1789-1808.

8. Basso C, Rizzo S, Valente M, et al: Cardiac masses and tumours. Heart 2016:102:1230-1245.

9. Pinede L, Duhaut P, Loire R: Clinical presentation of left atrial cardiac myxoma: A series of 112 consecutive cases. Medicine (Baltimore) 2001:80:159-172.

10. Cianciulli TF, Cozzarin A, Soumoulou JB, et al: Twenty years of clinical experience with cardiac myxomas: Diagnosis, treatment, and follow up. J Cardiovasc Imaging 2019:27:37-47.

11. Yu SH, Lim SH, Hong YS, et al: Clinical experiences of cardiac myxoma. Yonsei Med J 2006:47:367-371.

12. Bjessmo S, Ivert T: Cardiac myxoma: 40 years' experience in 63 patients. Ann Thorac Surg 1997:63:697700.

13. Grebenc ML, Rosado-De-Christenson ML, Green CE, et al: Cardiac myxoma: Imaging features in 83 patients. Radiographics 2002:22:673-689.

14. Zipes D, Libby P, Bonow R, et al: Braunwald's Heart Disease: A Textbook of Cardiovascular Medicine. Philadelphia, PA: Elsevier/Saunders; 2018.

15. Engberding R, Daniel WG, Erbel R, et al: Diagnosis of heart tumours by transoesophageal echocardiography: A multicentre study in 154 patients. Eur Heart J 1993:14:1223-1228.

16. Wintersperger BJ, Becker CR, Gulbins H, et al: Tumors of the cardiac valves: Imaging findings in magnetic resonance imaging, electron beam computed tomography, and echocardiography. Eur Radiol 2000:10:443-449.

17. Mendes GS, Abecasis J, Ferreira A, et al: Cardiac tumors: three decades of experience from a tertiary center: are we changing diagnostic work-up with new imaging tools? Cardiovasc Pathol 2020:49.

18. Hoey ETD, Mankad K, Puppala S, et al: MRI and CT appearances of cardiac tumours in adults. Clin Radiol 2009:64:1214-1230.

19. Pazos-López P, Pozo E, Siqueira ME, et al: Value of CMR for the differential diagnosis of cardiac masses. JACC Cardiovasc Imaging 2014:7:896-905.

20. Giusca S, Mereles D, Ochs A, et al: Incremental value of cardiac magnetic resonance for the evaluation of cardiac tumors in adults: experience of a high volume tertiary cardiology centre. Int J Cardiovasc Imaging 2017:33:879-888.

Table I. Preoperative echocardiographic characteristics of the cardiac myxoma (CM) and non-myxoma (NM) group

Echocardiographic

characteristics

$\mathrm{CM}(\mathrm{n}=45) \quad \mathrm{NM}(\mathrm{n}=8)$

Mean size (mm)

$35.3 \pm 18.6$ (range: $10-81$ )

$30.3 \pm 16.9$ (range: $8-50$ )

Location

Left atrium

$36(80)$

$2(25)$ 


\begin{tabular}{lll}
\hline $\begin{array}{l}\text { Echocardiographic } \\
\text { characteristics }\end{array}$ & $\mathrm{CM}(\mathrm{n}=45)$ & $\mathrm{NM}(\mathrm{n}=8)$ \\
\hline $\begin{array}{l}\text { Fossa ovalis } \\
\text { Mitral valve }\end{array}$ & $27(75)$ & $1(50)$ \\
Posterior interatrial septum & $4(11)$ & $1(50)$ \\
Free left atrial wall & $1(3)$ & $4(50)$ \\
Left atrial appendage & $1(3)$ & $1(12) 1(12)$ \\
Right atrium & $8(18)$ & $7(88) 1(12)$ \\
Left ventricle Aortic valve & $1(2)$ & \\
Mobility & $32(71) 5(11) 8(18)$ & $1(12)$ \\
Mobile Non-mobile No data & & $3(38) 4(50)$ \\
available & $28(62)$ & $2(25)$ \\
Surface & $8(18) 9(20)$ & $1(50)$ \\
Smooth or lobulated & $11(24)$ & $1(50)$ \\
Villous No data available & $10(21)$ & \\
$\begin{array}{l}\text { Obstruction (present) } \\
\text { Mitral valve }\end{array}$ & $1(9)$ & \\
Tricuspid valve & &
\end{tabular}

Values are presented as mean \pm standard deviation or number (percentage).

Table II. Comparison of demographic and echocardiographic characteristics between pathohistologically confirmed cardiac myxoma (CM) and non-myxoma (NM) groups.

\begin{tabular}{llll}
\hline Characteristic & $\mathrm{CM}(\mathrm{n}=39)$ & $\mathrm{NM}(\mathrm{n}=14)$ & $\mathrm{p}$ value \\
\hline Age (years) & $63.1 \pm 13.6$ & $66.6 \pm 15.1$ & 0.434 \\
Sex (female) & $25(64)$ & $10(71)$ & 0.620 \\
Location & & & $\mathrm{p}<0.001$ \\
Typical & $28(72)$ & 0 & $\mathrm{p}<0.001$ \\
Atypical & $11(28)$ & $14(100)$ & 0.017 \\
Size $(\mathrm{mm})$ & $37.9 \pm 18.3$ & $24.3 \pm 13.2$ & \\
\hline
\end{tabular}

Values are presented as mean \pm standard deviation or number (percentage).

\section{Hosted file}

image1.emf available at https://authorea.com/users/451885/articles/550079-accuracy-oftransthoracic-echocardiography-in-diagnosis-of-cardiac-myxoma-single-centre-experience

Fig.1 Panel A. Transthoracic echocardiography, apical 4-chamber view. Cardiac mass in left atrium is attached to the interatrial septum in the region of the fossa ovalis (arrow). Histopathological characterization confirmed cardiac myxoma. Panel B. Abundant myxoid stroma with clusters of myxoma cells forming cords and ring structures (HE 100x). RA - right atrium, RV - right ventricle, LA - left atrium, LV - left ventricle

\section{Hosted file}

image2.emf available at https://authorea.com/users/451885/articles/550079-accuracy-oftransthoracic-echocardiography-in-diagnosis-of-cardiac-myxoma-single-centre-experience

Fig.2 Flow diagram showing the diagnostic accuracy of preoperative TTE in patients with suspected CM. The predictive value and accuracy of preoperative TTE was calculated using the results of pathohistological 
examination as the gold standard. TP - true positive; FN - false negative; FP - false positive; TN - true negative; TTE - transthoracic echocardiography
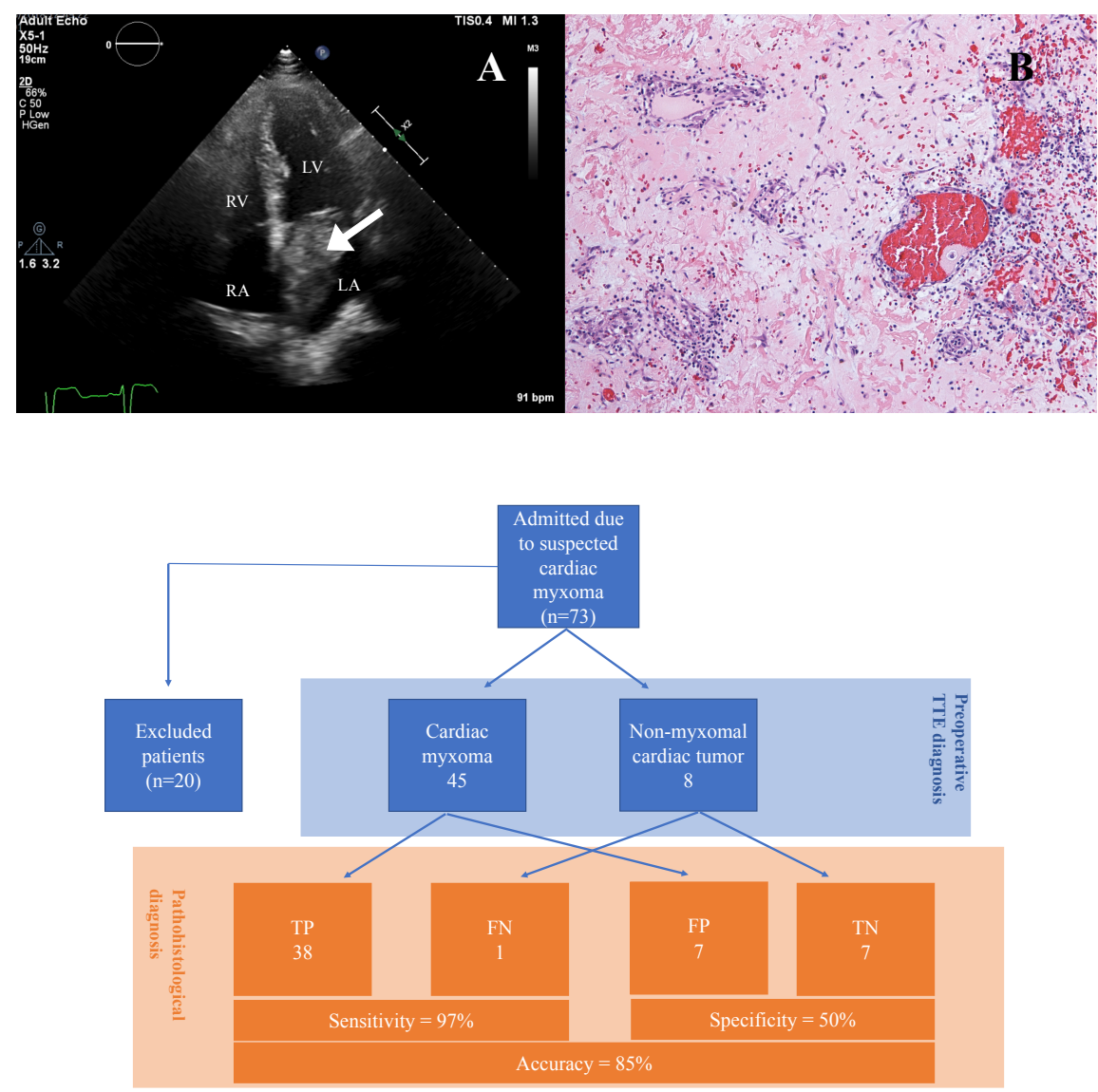